\title{
Editorial
}

\section{Too Many or Too Few Hands?}

\author{
Peter Heseltine, MD
}

In 1975, Haley investigated a series of neonatal infections at a large municipal hospital and determined that understaffing and overcrowding had contributed significantly to the outbreak. ${ }^{1}$ Almost 25 years later, though intuitive to infection control practitioners, surprisingly little controlled evidence exists to support this concept. The paper by Harbath et al in this issue identifies and measures the effects of understaffing, overcrowding, and handwashing on an outbreak of Enterobacter cloacae in a neonatal intensive care unit (ICU). ${ }^{2}$ The authors conclude that these factors had a primary role in sustaining the outbreak, which was only brought under control when they were changed.

Molecular epidemiology provides an opportunity to examine cross-infections in a scientific manner, and organisms such as $E$ cloacae lend themselves to this kind of investigation. ${ }^{3}$ Because most infection control practitioners, if not all physicians, are trained that hand washing is the single most important factor in the prevention of nosocomial infections, the facts are often fit to the hypothesis. Identical organisms can be tracked from patient to patient, observations are made of poor handwashing technique, and the conclusion is reached that these are cause and effect. Undoubtedly, cross-infections account for many nosocomial infections in ICUs. Almost all published reports of observations of hand washing by physicians and nurses lament their performance at this most basic of aseptic practices $^{4,5}$; but, if epidemiology is about measurement, where are there data that describe the magnitude of the effect that these practices (or their lack) have on the occurrence of epidemics?

If we are to assign time and resources to hand washing and isolation, should we not quantify how much of these is necessary to prevent an outbreak? If we cannot maintain an absolute standard of hand washing between each and every patient contact, what is a reasonable median that will achieve what we need? Are there differences among organisms in their capacity to be spread from patient to patient in this manner? What is the role of antibiotic prescribing in contributing to the colonization and so the reservoir of epidemic resistant organisms?

Modern ICUs present an environment remarkably well suited to the transmission of infection. Although patients are increasingly immunocompromised and thus susceptible to infection, many units were designed with inadequate space for all the equipment in current use and the procedures that are likely to occur. As an example, peritoneal lavage through an abdominal zipper is common in many surgical ICUs, affording an opportunity to contaminate much of the immediate environment with fecal organisms. When an epidemic is caused by an easily recognized organism such as a drug-resistant Salmonella species or Clostridium difficile, the contribution to the outbreak of the contaminated environment is easily recognized ${ }^{6,7}$; but, when the organisms causing infections are not so easily distinguished from "normal" ICU patient flora, the ease of contamination of one patient by another from fomites or secondary contamination of the staffs' hands may not be as obvious.

As is described for the University of Geneva Hospital's neonatal ICU, many units do not have separate rooms for each patient. It is worth noting, though, that at least one study found that this barrier did not reduce the frequency of cross-infection between patients, probably because contaminated equipment and hands easily pass from room to room. ${ }^{8}$ Still, units with designs that obstruct rather than facilitate hand decontamination and encourage contamination of the area and equipment around the patient are commonplace.

Control of serious outbreaks has sometimes required cohorting of staff between infected and noninfected patients. Although not detailed in his report, the neonatal ICU investigated by Haley resorted to separating physician and nursing staff who cared for infected patients from 
those caring for infants not infected, to control subsequent outbreaks. ${ }^{1}$ This is costly and not possible with limited staff. More commonly, isolation practices using single-use gowns, gloves, and sometimes masks, substitute for separating either patients or staff. Judging by the frequency with which cross-infections occur in ICUs, these practices are not very effective. Yet, increased isolation strategies are employed routinely in outbreak situations in ICUs, sometimes with effect, sometimes without, but always at cost and inconvenience.

What then are the steps that we should consider to limit cross-infections in ICUs as modern surgery enters into a second century and as neonatal ICUs turn 50 ? Should we be content with the old concepts and "just do it" better?

Most of us do not have the opportunity to redesign our ICUs and correct the problems that often become obvious only when the ICUs are in use. Although these design flaws are often well known to the staff, "work-arounds" that are possible are rarely considered until outbreaks have occurred. Surely part of the role of an infection control committee is to participate in the design and monitor the safe operation of an ICU. ${ }^{9}$ How many receive information, as part of their standard monthly reports, on staffing and work-load patterns that might enable them to alert the hospital to an impending outbreak or a potentially dangerous situation? Can it be that the medical staff is shielded from this information to avoid discussion of the impact of costcutting measures?

Basic standards and statistics that reflect the operation of an ICU are an essential part of its safe management. Conditions that promote cross-infections are no less worthy of consideration than faulty electrical wiring or unsafe working conditions. Just as the monthly infection statistics are noted, so should work load and staffing indicators be considered.

Microbiology results are commonly reported to the infection control personnel and committee responsible for oversight of most ICUs. Tabulations of the organisms causing infections and their antibiotic susceptibilities are reported routinely. From these data determinations of cross-infections are often inferred. But what of the contribution of antibiotic prescribing by physicians? Harbath and colleagues comment that two specific antibiotics are selected routinely for suspected sepsis in their hospital. Evans and Pestotnik have observed that the selection of the same antibiotics for all presumed infections is likely to lead to the emergence of drug-resistant colonizations and infections due to selective pressure on patients' gastrointestinal and respiratory flora. ${ }^{10,11}$ This selection practice often is reinforced by restricted hospital formularies. Recent recommendations curtailing the use of vancomycin as a control measure against vancomycin-resistant enterococci (VRE) demonstrate the importance of this strategy in control of other pathogens. Cross-infections with resistant organisms have to start somewhere. Some patients may harbor small numbers of resistant organisms and, through treatment with broad spectrum antibiotics and consequent overgrowth, increase their numbers and become important reservoirs of these pathogens for other patients.
The control of cross-infections and antibiotic-resistant pathogens is not in fact about one strategy but about several. Hand washing alone does not work. If it did, no other strategy would be needed to deal with outbreaks.

Isolation alone does not work for similar empirical reasons. None of these control measures, including antibiotic restrictions, works when applied exclusively after the fact, when the problem becomes epidemic, for the seeds of the matter are endemic and always present.

Most hospitals admit patients from other institutions. This is a function of the integration of specialty care. The sickest patients often are admitted to the most crowded units, and trained staff and sufficient space are always in short supply. For 50 years, the physician's response to infection has been to use increasingly powerful antibiotics. This sequence is the real cause and effect of crossinfections. The State of California recently required hospitals to assure that their medical staff adhere to infection control practices. In response, many institutions have begun to observe and record if doctors and nurses wash their hands before and after handling patients. Intensive-care units are a frequent site for this monitoring because of the generally accepted risk of cross-infections in this environment. Initial statistics are disappointing but not unexpected. Nurses wash their hands about twice as often as physicians, but still fail to do so approximately $35 \%$ of the time. This frequency does not seem sufficient to prevent cross-infection with methicillinresistant Staphylococcus aureus (MRSA) or drug-resistant gram-negative Enterobacteriaceae (extended-spectrum $\beta$ lactamases [ESBLs]) when prevalent in an ICU setting. During outbreaks, when all staff are achieving a rate closer to $90 \%$, transmission of MRSA and some gram-negatives can be stopped; however, polyclonal outbreaks of ESBLs, organisms not directly related to each other, are largely unaffected by this strategy, as are VRE. The latter are much more likely to be controlled by changes in antibiotic prescribing patterns.

These empirical and preliminary findings suggest that many outbreaks are truly the tip of the iceberg and that cross-infection control programs need to draw on several simultaneous strategies, tailored to the information derived from the local situation. If we are to create a safer house for newborns or ICU patients, that house needs not merely a single wall of protection, good hand washing, but three others, too: control of fomites and cross-contamination of the environment, the proper evidence-based prescribing of antibiotics, and knowledge of work-load, supply, and staffing issues that permit an oversight group to change in real time the conditions that threaten the patients, not just respond when the epidemic curve is obvious.

\section{REFERENCES}

1. Haley RW, Bregman DA. The role of understaffing and overcrowding in recurrent outbreaks of staphylococcal infection in a neonatal special care unit. I Infect Dis 1982;145:875-885.

2. Harbarth S, Sudre P, Dharan S, Cadenas M, Pittet D. Outbreak of Enterobacter cloacae related to understaffing, overcrowding, and poor hygiene practices. Infect Control Hosp Epidemiol 1999;20:598-603.

3. Hacek DM, Suriano T, Noskin GA, Kruszynski J, Reisberg B, Peterson LR. Medical and economic benefit of a comprehensive infection control 
program that includes routine determination of microbial clonality. $A m \mathrm{~J}$ Clin Pathol 1999:111:647-654.

4. Bittner MJ, Rich EC. Surveillance of handwashing episodes in adult intensive-care units by measuring an index of soap and paper towel consumption. Clinical Performance and Quality Health Care 1998;6:179-182.

5. Watanakunakorn C, Wang C, Hazy J. An observational study of hand washing and infection control practices by healthcare workers. Infect Control Hosp Epidemiol 1998;19:858-860.

6. Revathi G, Shannon KP, Stapleton PD, Jain BK, French GL. An outbreak of extended-spectrum, beta-lactamase-producing Salmonella senftenberg in a burns ward. $J$ Hosp Infect 1998;40:295-302

7. Struelens MJ, Maas A, Nonhoff C, Deplano A, Rost F, Serruys E, et al. Control of nosocomial transmission of Clostridium difficile based on spo- radic case surveillance. Am J Med 1991;91:138S-144S.

8. Kerr JR, Moore JE, Curran MD, Graham R, Webb CH, Lowry KG, et al. Investigation of a nosocomial outbreak of Pseudomonas aeruginosa pneumonia in an intensive care unit by random amplification of polymorphic DNA assay. J Hosp Infect 1995;30:125-131.

9. Harvey MA. Critical-care-unit bedside design and furnishing: impact on nosocomial infections. Infect Control Hosp Epidemiol 1998;19:597-601.

10. Evans RS, Pestotnik SL, Classen DC, Clemmer TP, Weaver LK, Orme JF Ir, et al. A computer-assisted management program for antibiotics and other antiinfective agents. $N$ Engl J Med 1998;338:232-238.

11. Pestotnik SL, Classen DC, Evans RS, Burke JP. Implementing antibiotic practice guidelines through computer-assisted decision support: clinical and financial outcomes. Ann Intern Med 1996;124:884-890. 